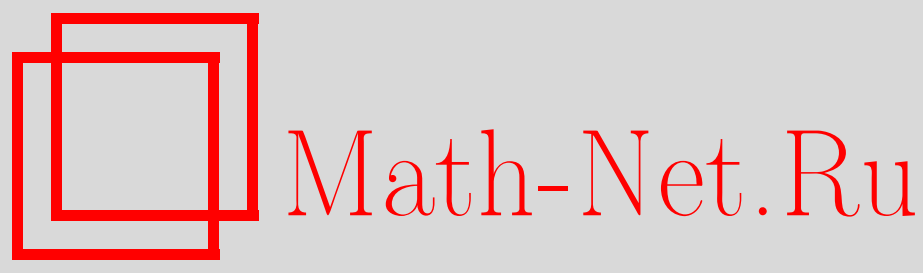

Р. С. Нестеренко, Уточнение определения гравитационной энергии, ТМФ, 2010, том 162, номер 1, 150-160

DOI: https://doi.org/10.4213/tmf6461

Использование Общероссийского математического портала Math-Net.Ru подразумевает, что вы прочитали и согласны с пользовательским соглашением http://www . mathnet.ru/rus/agreement

Параметры загрузки:

IP: 54.172 .240 .79

26 апреля 2023 г., 13:11:03

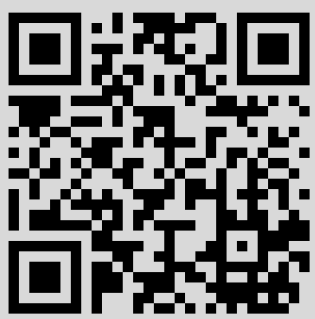




\section{УТОЧНЕНИЕ ОПРЕДЕЛЕНИЯ ГРАВИТАЦИОННОЙ ЭНЕРГИИ}

Погрешность известного "энергетического" метода вычисления центральной плотности белого карлика с предельно возможной массой анализируется в рамках теории тетрадных комплексов энергии-импульса гравитационного поля. Путем сравнения величины центральной плотности, вычисленной с помощью каждого из трех рассмотренных комплексов, с ее значением, полученным с помощью численного интегрирования уравнений Эйнштейна, производится выбор преимущественного комплекса.

Ключевые слова: гравитационная энергия, тетрадный комплекс, предел Чандрасекара.

\section{1. ВВЕДЕНИЕ}

Проблема гравитационной энергии возникла вместе с появлением общей теории относительности. Для решения этой проблемы предлагались различные псевдотензоры, комплексы энергии-импульса и т.д. Усилия исследователей были направлены на выделение преимущественного псевдотензора/комплекса. Всякий раз оказывалось, что выбором системы координат/системы отсчета можно получить на их основе самые нелепые результаты. На наш взгляд, выбор между величинами, характеризующими гравитационную энергию, невозможно осуществить без привязки теоретических рассуждений к непробному веществу. Поэтому мы рассмотрим как можно более самосогласованную задачу, в которой материя взаимодействует с создаваемым ею гравитационным полем. Для наших целей подходящей является задача о пределе Чандрасекара для белых карликов.

Сразу отбросив псевдотензорный подход как зависящий от системы координат и затрудняющий физическую интерпретацию получаемых результатов в определенной системе отсчета, мы сосредоточимся на формализме тетрадных комплексов. Ориентируясь на книги [1], [2], будем искать приемлемый тетрадный комплекс энергии-импульса гравитационного поля путем сравнения результатов с точным методом интегрирования уравнений Эйнштейна (в которых гравитационная энергия уже учтена).

*E-mail: rs-nesterenko@mail.ru 


\section{2. СУТЬ ЭНЕРГЕТИЧЕСКОГО МЕТОДА}

В этом разделе мы используем геометрические единицы $c=G=1$.

В рамках энергетического метода считается [1], [2], что величина полной энергии есть сумма следующих слагаемых:

$$
E_{\mathrm{int}}+E_{\mathrm{GravNewt}}+\Delta E_{\mathrm{int}}+\Delta E_{\mathrm{GTR}}
$$

где $E_{\mathrm{int}}-$ внутренняя энергия звезды, $E_{\mathrm{GravNewt}}$ - ньютоновская гравитационная энергия, $\Delta E_{\mathrm{int}}$ - поправка, обусловленная отличием уравнения состояния от политропы с $n=3$. Поправка общей теории относительности $\Delta E_{\mathrm{GTR}}$ вычисляется путем разложения в ряд "энергии звезды" (за вычетом энергии покоя)

$$
E_{\mathrm{GTR}}=\int_{0}^{R}\left[\rho\left(1-\frac{2 m}{r}\right)^{1 / 2}-\rho_{0}\right] d V \approx \int_{0}^{R} \rho_{0}\left[u-\frac{m}{r}-u \frac{m}{r}-\frac{1}{2}\left(\frac{m}{r}\right)^{2}\right] d V
$$

и вычитания из этого выражения ньютоновской энергии

$$
E_{\mathrm{Newt}}=\int_{0}^{R} \rho_{0} u d V-\int_{0}^{M} \frac{m^{\prime}}{r^{\prime}} d m^{\prime} .
$$

Здесь

$r^{\prime}=\left(\frac{3 V}{4 \pi}\right)^{1 / 3}, \quad \rho=\rho_{0}(1+u), \quad d m=\rho 4 \pi r^{2} d r, \quad d m^{\prime}=\rho_{0} d V, \quad \rho_{0}=m_{\mathrm{B}} n$,

где $m_{\mathrm{B}}$ - масса бариона, $n$ - концентрация барионов, $d V=(1-2 m / r)^{-1 / 2} 4 \pi r^{2} d r-$ элемент собственного объема геометрии Шварцшильда, $u$ - внутренняя энергия на единицу массы. Считая, что $\rho_{0}(V)$ - одна и та же функция и в теории Ньютона, и в общей теории относительности (ОТО), в книгах [1], [2] авторы сокращают член $\rho_{0} u$ и далее рассматривают оставшиеся члены.

Энергетический метод в такой форме дает значение $\rho_{\mathrm{c}}=2.635 \cdot 10^{10} \mathrm{\Gamma} / \mathrm{cm}^{3}$ для центральной плотности белого карлика предельной массы (авторы монографии [1] использовали прежние значения фундаментальных констант, и у них $\rho_{\mathrm{c}}=$ $\left.=2.646 \cdot 10^{10} \mathrm{\Gamma} / \mathrm{cm}^{3}\right)$. Во всех расчетах мы принимаем, что отношение числа барионов к числу электронов $\mu_{\mathrm{e}}=2$.

\section{3. ИНТЕГРИРОВАНИЕ УРАВНЕНИЙ ВНУТРЕННЕГО СТРОЕНИЯ ЗВЕЗДЫ}

В этом разделе мы приводим сводку результатов численных расчетов, алгоритм которых заключается в выборе невращающихся сферически-симметричных звездных конфигураций, обладающих наибольшей массой при заданных условиях. Параметром моделей звезд можно считать центральную плотность $\rho_{\mathrm{c}}$.

Для расчета внутреннего строения сферически-симметричной статической звезды необходимо проинтегрировать уравнения Толмана-Оппенгеймера-Волкова (ТОВ)

$$
\frac{d m}{d r}=4 \pi r^{2} \rho, \quad \frac{d P}{d r}=-\frac{\left(\rho c^{2}+P\right)\left(G m / c^{2}+4 \pi G r^{3} P / c^{4}\right)}{r\left(r-2 G m / c^{2}\right)},
$$


являющиеся следствиями уравнений Эйнштейна [3]. В ньютоновской теории гравитации уравнение гидростатического равновесия упрощается и принимает вид

$$
\frac{d P}{d r}=-\frac{G m}{r^{2}} \rho
$$

Для интегрирования уравнений внутреннего строения звезды необходимо задать уравнение состояния. Мы произвели расчеты для следующих случаев.

СлучАй 1. Близкое к точному уравнение состояния + численное интегрирование уравнений ТОВ:

$$
\begin{aligned}
& P=P_{\mathrm{e}}(x)=\frac{\pi m_{\mathrm{e}}^{4} c^{5}}{h^{3}}\left[x\left(\frac{2 x^{2}}{3}-1\right) \sqrt{x^{2}+1}+\ln \left(x+\sqrt{x^{2}+1}\right)\right], \\
& \rho=\rho_{\text {ion }}(x)=\mu_{\mathrm{e}} m_{\mathrm{u}} n_{\mathrm{e}}=\mu_{\mathrm{e}} m_{\mathrm{u}} \frac{8 \pi}{3}\left(\frac{m_{\mathrm{e}} c}{h}\right)^{3} x^{3}, \quad x=\frac{p_{\mathrm{F}}}{m_{\mathrm{e}} c}
\end{aligned}
$$

где $m_{\mathrm{u}}$ - атомная единица массы, $x$ - релятивистский параметр, $h$ - постоянная Планка, $m_{\mathrm{e}}$ - масса электрона, $p_{\mathrm{F}}$ - импульс Ферми. Газ электронов полностью вырожден, давление определяется электронами, вклад в плотность дает масса покоя ионов. Для $\mu_{\mathrm{e}}=2$ получаем следующие значения максимальной массы и соответствующих ей центральной плотности и радиуса белого карлика:

$$
M_{\mathrm{WD}}=1.4254103 M_{\odot}, \quad \rho_{\mathrm{c}}=3.555 \cdot 10^{10}{ }_{\mathrm{r}} / \mathrm{cm}^{3}, \quad R=9.0504 \cdot 10^{7} \mathrm{~cm} .
$$

СлУчАй 2. Политропное уравнение состояния с $n=3+$ уравнения ТОВ:

$$
P=K \rho^{1+1 / n}, \quad K=\frac{1}{8}\left(\frac{3}{\pi}\right)^{1 / 3} \frac{c h}{\left(m_{\mathrm{u}} \mu_{\mathrm{e}}\right)^{4 / 3}}
$$

или

$$
P \approx \frac{\pi m_{\mathrm{e}}^{4} c^{5}}{h^{3}} \frac{2 x^{4}}{3}, \quad \rho=\rho_{\text {ion }}(x) .
$$

Уравнение состояния ультрарелятивистского вырожденного электронного газа соответствует политропе индекса $n=3$. Для этих эквивалентных случаев получаем, что кривая $M\left(\rho_{\mathrm{c}}\right)$ не имеет максимума, масса $M$ монотонно убывает с ростом $\rho_{\mathrm{c}}$.

СлУчАй 3. Политропное уравнение состояния с поправками + уравнения ТОВ:

$$
P \approx \frac{2 \pi m_{\mathrm{e}}^{4} c^{5}}{3 h^{3}}\left(x^{4}-x^{2}\right), \quad \rho=\rho_{\text {ion }}(x) .
$$

Уже при учете первой поправки к политропному уравнению состояния появляется максимум на кривой $M\left(\rho_{\mathrm{c}}\right)$. Результаты случаев 2 и 3 показывают, что в рамках энергетического метода следует учитывать поправки к уравнению состояния: $E=$ $E_{\text {grav }}+E_{\text {int }}+\Delta E_{\text {grav }}+\Delta E_{\text {int }}$, отбрасывать $\Delta E_{\text {int }}$ нельзя.

СлУчАй 4. Политропное уравнение состояния с $n=3+$ ньютоновская теория гравитации. В этом случае масса конфигурации не зависит от центральной плотности, и ее численное значение $M_{\mathrm{Ch}}=1.45635 M_{\odot}$ соответствует аналитической 
теории, в которой

$$
M_{\mathrm{Ch}}=\frac{4 K^{3 / 2}}{G^{3 / 2} \sqrt{\pi}} \int_{0}^{\xi_{1}} \theta^{3} \xi^{2} d \xi=1.45631 M_{\odot}
$$

Случай 5. Близкое к точному уравнение состояния $P=P_{\mathrm{e}}(x), \rho=\rho_{\text {ion }}(x)$ + ньютоновская теория гравитации. При увеличении $x$ соотношение между $P$ и $\rho$ стремится к политропному уравнению состояния с $n=3$, поэтому масса должна стремиться к пределу Чандрасекара. И это действительно так, что можно увидеть из табл. 1.

ТАБЛИцА 1

\begin{tabular}{|c|c|c|c|c|}
\hline$x_{\mathrm{c}}$ & $\rho_{\mathrm{c}}, \Gamma / \mathrm{cM}^{3}$ & $P_{\mathrm{c}}, \Gamma / \mathrm{cM} \cdot \mathrm{c}^{2}$ & $M_{\mathrm{WD}}, M_{\odot}$ & $R_{\mathrm{WD}}, \mathrm{KM}$ \\
\hline 10 & $1.948 \cdot 10^{9}$ & $1.189 \cdot 10^{27}$ & 1.394728 & $2.073 \cdot 10^{3}$ \\
$10^{2}$ & $1.948 \cdot 10^{12}$ & $1.200 \cdot 10^{31}$ & 1.455556 & $2.592 \cdot 10^{2}$ \\
$10^{3}$ & $1.948 \cdot 10^{15}$ & $1.200 \cdot 10^{35}$ & 1.456340 & 26.70 \\
$10^{4}$ & $1.948 \cdot 10^{18}$ & $1.200 \cdot 10^{39}$ & 1.456348 & 2.679 \\
$10^{5}$ & $1.948 \cdot 10^{21}$ & $1.200 \cdot 10^{43}$ & 1.456348 & 0.2681 \\
\hline
\end{tabular}

Можно также сделать вывод, что точность вычислений достаточна для наших целей.

\section{4. ОПИСАНИЕ МОДИФИЦИРОВАННОГО ЭНЕРГЕТИЧЕСКОГО МЕТОДА}

Наша модификация энергетического метода заключается в том, что мы рассматриваем величины, заведомо сохраняющиеся в ОТО. Общая схема построения тетрадных комплексов энергии-импульса гравитационного поля заключается в следующем. Уравнения Эйнштейна записываются в полутетрадном виде [4]

$$
\frac{g_{\nu}(\alpha)}{\kappa}\left(R^{\mu \nu}-\frac{1}{2} g^{\mu \nu} R\right)=\theta^{\mu}(\alpha)-t^{\mu}(\alpha)=P^{\mu}(\alpha), \quad P^{\mu}(\alpha)=T^{\mu \nu} g_{\nu}(\alpha)
$$

где $g_{\nu}(\alpha)$ - компоненты тетрады, $\alpha$ - лоренцев индекс, $\nu$ - координатный индекс, $T^{\mu \nu}$ - тензор энергии-импульса вещества. Векторы $t^{\mu}(\alpha)$ составляют так называемый тетрадный комплекс энергии-импульса гравитационного поля. Существуют законы сохранения

$$
\begin{gathered}
\nabla_{\mu} \theta^{\mu}(\alpha)=\nabla_{\mu}\left(P^{\mu}(\alpha)+t^{\mu}(\alpha)\right)=\frac{1}{\sqrt{-g}} \partial_{\mu}\left[\sqrt{-g}\left(P^{\mu}(\alpha)+t^{\mu}(\alpha)\right)\right]=0 \\
P(\alpha)=\int \sqrt{-g}\left[P^{\mu}(\alpha)+t^{\mu}(\alpha)\right] d \sigma_{\mu} .
\end{gathered}
$$

Интеграл для $\alpha=0$, как мы увидим ниже, дает полную энергию звезды, состоящую из внутренней энергии газа, энергии покоя и гравитационной энергии. 
Для сферически-симметричной звезды линейный элемент выражается формулой

$$
d s^{2}=-e^{2 \Phi / c^{2}} d t^{2} c^{2}+\frac{d r^{2}}{1-2 G m / c^{2} r}+r^{2}\left(d \theta^{2}+\sin ^{2} \theta d \phi^{2}\right),
$$

таким образом, для $\alpha=0$ правая часть (8) переходит в

$$
\int_{0}^{R} \theta^{0}(0) g_{0}(0) \frac{4 \pi r^{2} d r}{\sqrt{1-2 G m / c^{2} r}}
$$

где $R$ - радиус звезды.

Опишем дальнейшие преобразования этого интеграла, которые отвечают преобразованиям, используемым в "энергетическом" методе [1], [2]. Разлагая этот интеграл в ряд и "обезразмеривая" с помощью формул теории политроп $(\theta(\xi)-$ функция Эмдена, $\xi_{1}$ - корень функции Эмдена),

$$
\begin{gathered}
\Phi=\Phi_{\mathrm{c}} \theta(\xi), \quad \Phi_{\mathrm{c}}=(n+1) K \rho_{\mathrm{c}}^{1 / n}, \\
\rho_{0}=\rho_{\mathrm{c}} \theta^{n}, \quad r=r_{1} \xi, \quad r_{1}=\frac{(n+1)^{1 / 2} K^{1 / 2}}{(4 \pi G)^{1 / 2}} \rho_{\mathrm{c}}^{1 / 2 n-1 / 2}, \quad n=3, \quad \\
\theta^{\prime \prime}=-\theta^{n}-\frac{2}{\xi} \theta^{\prime}, \quad m(r)=-4 \pi r_{1}^{3} \rho_{\mathrm{c}} \xi^{2} \theta^{\prime}, \quad m^{\prime}(r)=4 \pi r_{1}^{3} \rho_{\mathrm{c}} \theta^{n} \xi^{2}, \quad R=r_{1} \xi_{1},
\end{gathered}
$$

мы получаем выражение для полной энергии, вычисленной с помощью какого-либо тетрадного комплекса:

$$
A \rho_{\mathrm{c}}^{1 / 3}+B \rho_{\mathrm{c}}^{2 / 3}+C \rho_{\mathrm{c}}^{-1 / 3}+D=E .
$$

Равновесие достигается при $\partial E / \partial \rho_{\mathrm{c}}=0$, устойчивость нарушается при $\partial^{2} E / \partial \rho_{\mathrm{c}}^{2}=0$, что в явном виде записывается как

$$
\begin{aligned}
\frac{1}{3} A \rho_{\mathrm{c}}^{-2 / 3}+\frac{2}{3} B \rho_{\mathrm{c}}^{-1 / 3}-\frac{1}{3} C \rho_{\mathrm{c}}^{-4 / 3} & =0, \\
-\frac{2}{9} A \rho_{\mathrm{c}}^{-5 / 3}-\frac{2}{9} B \rho_{\mathrm{c}}^{-4 / 3}+\frac{4}{9} C \rho_{\mathrm{c}}^{-7 / 3} & =0 .
\end{aligned}
$$

Следуя книге [1], выражаем коэффициент при $\rho_{\mathrm{c}}^{-2 / 3}$ из первого уравнения и подставляем во второе уравнение, но, в отличие от [1], мы не будем искусственно выделять массу $M$ в коэффициентах разложения ${ }^{1)}$. После этого мы получаем одно уравнение для $\rho_{\mathrm{c}}$, из которого следует формула

$$
\rho_{\mathrm{c}}=-\frac{C}{B}
$$

1) Авторы книги [1] действуют полностью эквивалентным образом: сначала выделяют массу $M$ по формуле из теории политроп, а затем, после подстановки коэффициента при $\rho_{\mathrm{c}}^{-2 / 3}$ во второе уравнение, заменяют $M$ обратно на ее политропное значение. 


\section{5. ТЕТРАДНЫЙ КОМПЛЕКС ФРОЛОВА}

Комплекс Фролова [4] определяется следующими сохраняющимися векторами:

$$
\theta^{\mu}(\alpha)=\frac{2}{\kappa} \nabla_{\nu} C^{\mu \nu},
$$

где $C_{\mu \nu}=\left(\partial_{\mu} g_{\nu}(\alpha)-\partial_{\nu} g_{\mu}(\alpha)\right) / 2$ - объекты неголономности (в книге [4] неточность: приведено выражение $(13)$ для $\theta^{\mu}(\alpha)$, но векторы обозначены как $t^{\mu}(\alpha)$, хотя эти величины совпадают только в отсутствие непробного вещества).

Вычислим плотность гравитационной энергии для статической сферически-симметричной метрики. Имеем

$$
S_{00}=g_{\mu}(0) \theta^{\mu}(0)=g_{0}(0) \theta^{0}(0)=g_{0}(0) \frac{1}{\kappa} \frac{1}{\sqrt{-g}} \partial_{1}\left[\sqrt{-g} g^{00} g^{11}\left(-\partial_{1} g_{0}(0)\right)\right] .
$$

Для метрики Шварцшильда

$$
S_{00}(\mathrm{Schw})=-\frac{G m^{2}}{8 \pi r^{4}\left(1-2 G m / c^{2} r\right)},
$$

что соответствует ньютоновскому значению с поправкой, связанной с замедлением времени.

Для расчета критической центральной плотности белого карлика нужно вычислить интеграл

$$
\begin{aligned}
\int_{0}^{R} & S_{00} \frac{4 \pi r^{2} d r}{\sqrt{1-2 G m / c^{2} r}} \approx 4 \pi \int_{0}^{R} r^{2} S_{00}\left(1+\frac{G m}{c^{2} r}+\frac{3 G^{2} m^{2}}{2 c^{4} r^{2}}\right) d r \approx \\
& \approx \int_{0}^{R}\left[-\frac{m^{2} \Phi^{\prime \prime} G}{4 c^{2}}-\frac{m m^{\prime} \Phi^{\prime} G}{2 c^{2}}+\frac{c^{2} r^{2} \Phi^{\prime \prime}}{2 G}+\frac{c^{2} r \Phi^{\prime}}{G}-\frac{r m \Phi^{\prime \prime}}{2}-\frac{r m^{\prime} \Phi^{\prime}}{2}-\frac{m \Phi^{\prime}}{2}\right] d r
\end{aligned}
$$

Здесь $S_{00}$ вычисляется по формуле (14) при соответствующих значениях компонент тетрады и метрики. Используя безразмерные величины, заданные с помощью формул (10), с точностью до членов порядка $m^{3} / r^{3}$ будем иметь в правой части последнего равенства следующее выражение:

$$
\begin{aligned}
& -\frac{8 \rho_{\mathrm{c}}^{1 / 3} K^{5 / 2} \sqrt{\pi G}}{\pi G^{2}} \int_{0}^{\xi_{1}} \xi^{2}\left(\theta^{\prime}\right)^{2} d \xi-\frac{8 K^{3}}{\pi G^{2}} \int_{0}^{\xi_{1}} \xi^{3} \theta^{3} \theta^{\prime} d \xi- \\
& -\frac{8 \rho_{\mathrm{c}}^{1 / 3} K^{5 / 2} \sqrt{\pi G}}{\pi G^{2}} \int_{0}^{\xi_{1}} \xi^{3} \theta^{3} \theta^{\prime} d \xi-\frac{2 c^{2} K^{3 / 2} \sqrt{\pi G}}{\pi G^{2}} \int_{0}^{\xi_{1}} \xi^{2} \theta^{3} d \xi+ \\
& +\frac{32 \rho_{\mathrm{c}}^{2 / 3} K^{7 / 2} \sqrt{\pi G}}{c^{2} \pi G^{2}} \int_{0}^{\xi_{1}} \xi^{3}\left(\theta^{\prime}\right)^{3} d \xi+\frac{32 \rho_{\mathrm{c}}^{1 / 3} K^{4}}{c^{2} \pi G^{2}} \int_{0}^{\xi_{1}} \xi^{4} \theta^{3}\left(\theta^{\prime}\right)^{2} d \xi+ \\
& +\frac{16 \rho_{c}^{2 / 3} K^{7 / 2} \sqrt{\pi G}}{c^{2} \pi G^{2}} \int_{0}^{\xi_{1}} \xi^{4} \theta^{3}\left(\theta^{\prime}\right)^{2} d \xi .
\end{aligned}
$$


Вычислим коэффициенты при различных степенях $\rho_{\text {c }}$ в выражении для полной энергии $A_{\Phi} \rho_{\mathrm{c}}^{1 / 3}+B_{\Phi} \rho_{\mathrm{c}}^{2 / 3}+D_{\Phi}$ :

$$
A_{\Phi}=6.421731 \cdot 10^{52}, \quad B_{\Phi}=-1.054929 \cdot 10^{42}, \quad D_{\Phi}=6.088957 \cdot 10^{58} .
$$

\section{6. ТЕТРАДНЫЙ КОМПЛЕКС РОДИЧЕВА}

Сохраняющиеся векторы для тетрадного комплекса Родичева [4] записываются каK

$$
\theta^{\mu}(\alpha)=\frac{2}{\kappa} \nabla_{\nu} \Delta(\alpha)^{\mu \nu}
$$

где $\Delta(\alpha)^{\mu \nu}=g^{\sigma}(\alpha) \Delta_{\sigma}^{\mu \nu}-$ коэффициенты вращения Риччи. Для $\Delta_{\mu, \alpha \nu}$ есть явное выражение [4]:

$$
\begin{aligned}
\Delta_{\mu, \alpha \nu}= & \frac{g_{\nu}(\beta)\left[\partial_{\alpha} g_{\mu}(\beta)-\partial_{\mu} g_{\alpha}(\beta)\right]}{2}+\frac{g_{\alpha}(\beta)\left[\partial_{\mu} g_{\nu}(\beta)-\partial_{\nu} g_{\mu}(\beta)\right]}{2}+ \\
& +\frac{g_{\mu}(\beta)\left[\partial_{\alpha} g_{\nu}(\beta)-\partial_{\nu} g_{\alpha}(\beta)\right]}{2}
\end{aligned}
$$

Плотность энергии для сферически-симметричной статической метрики равна

$$
S_{00}=g_{\mu}(0) \theta^{\mu}(0)=g_{0}(0) \theta^{0}(0)=-\frac{2}{\kappa} g_{0}(0) \frac{1}{\sqrt{-g}} \partial_{1}\left[\sqrt{-g} g_{0}^{2}(0)\left(g^{00}\right)^{2} g^{11} \partial_{1} g_{0}(0)\right]
$$

Для метрики и тетрады Шварцшильда получаем

$$
S_{00}(\mathrm{Schw})=\frac{G m^{2}}{4 \pi r^{4}\left(1-2 G m / c^{2} r\right)} .
$$

Для сферически-симметричной звезды имеем

$$
\begin{aligned}
\int_{0}^{R} S_{00} \frac{4 \pi r^{2} d r}{\sqrt{1-2 G m / c^{2} r}} \approx & \int_{0}^{R}\left[\frac{m^{2} \Phi^{\prime \prime} G}{2 c^{2}}+\frac{m m^{\prime} \Phi^{\prime} G}{c^{2}}-\frac{c^{2} r^{2} \Phi^{\prime \prime}}{G}-\right. \\
& \left.-\frac{2 c^{2} r \Phi^{\prime}}{G}+r m \Phi^{\prime \prime}+r m^{\prime} \Phi^{\prime}+m \Phi^{\prime}\right] d r .
\end{aligned}
$$

Используя безразмерные величины, получаем в правой части последнего равенства

$$
\begin{aligned}
& -\frac{64 \rho_{\mathrm{c}}^{2 / 3} K^{7 / 2} \sqrt{\pi G}}{c^{2} \pi G^{2}} \int_{0}^{\xi_{1}} \xi^{3}\left(\theta^{\prime}\right)^{3} d \xi-\frac{64 \rho_{\mathrm{c}}^{1 / 3} K^{4}}{c^{2} \pi G^{2}} \int_{0}^{\xi_{1}} \xi^{4} \theta^{3}\left(\theta^{\prime}\right)^{2} d \xi- \\
& -\frac{32 \rho_{\mathrm{c}}^{2 / 3} K^{7 / 2} \sqrt{\pi G}}{c^{2} \pi G^{2}} \int_{0}^{\xi_{1}} \xi^{4} \theta^{3}\left(\theta^{\prime}\right)^{2} d \xi+\frac{16 \rho_{\mathrm{c}}^{1 / 3} K^{5 / 2} \sqrt{\pi G}}{\pi G^{2}} \int_{0}^{\xi_{1}} \xi^{2}\left(\theta^{\prime}\right)^{2} d \xi+ \\
& +\frac{16 K^{3}}{\pi G^{2}} \int_{0}^{\xi_{1}} \xi^{3} \theta^{3} \theta^{\prime} d \xi+\frac{16 \rho_{\mathrm{c}}^{1 / 3} K^{5 / 2} \sqrt{\pi G}}{\pi G^{2}} \int_{0}^{\xi_{1}} \xi^{3} \theta^{3} \theta^{\prime} d \xi+\frac{4 c^{2} K^{3 / 2} \sqrt{\pi G}}{\pi G^{2}} \int_{0}^{\xi_{1}} \xi^{2} \theta^{3} d \xi .
\end{aligned}
$$

Последнее слагаемое представляет собой энергию покоя $M c^{2}$ для политропы с $n=3$.

Вычислим коэффициенты при различных степенях $\rho_{c}$ в выражении для полной энергии $A_{\mathrm{P}} \rho_{\mathrm{c}}^{1 / 3}+B_{\mathrm{P}} \rho_{\mathrm{c}}^{2 / 3}+D_{\mathrm{P}}$ :

$$
A_{\mathrm{P}}=-1.284346 \cdot 10^{53}, \quad B_{\mathrm{P}}=2.109857 \cdot 10^{42}, \quad D_{\mathrm{P}}=-1.217791 \cdot 10^{59} .
$$




\section{7. ПРЕИМУЩЕСТВЕННЫЙ ТЕТРАДНЫЙ КОМПЛЕКС}

В этом разделе мы будем использовать обозначения $e_{a \mu}=g_{\mu}(a), a=0,1,2,3$, $\mu=0,1,2,3$.

Для вывода данного комплекса энергии-импульса уравнения Эйнштейна необходимо представить в полутетрадной форме

$$
\begin{aligned}
G_{\mu \nu} & =\kappa T_{\mu \nu}, \quad G_{a \mu}=e_{a}^{\nu} G_{\mu \nu}=\kappa T_{a \mu} \\
g_{\mu \nu} & =\eta^{a b} e_{a \mu} e_{b \nu}=-e_{0 \mu} e_{0 \nu}+e_{1 \mu} e_{1 \nu}+e_{2 \mu} e_{2 \nu}+e_{3 \mu} e_{3 \nu}
\end{aligned}
$$

Переписывая эти уравнения в виде

$$
\mathbf{R}_{a}=R_{a \nu} \mathbf{d} x^{\nu}=\kappa\left(\mathbf{T}_{a}-\frac{1}{2} T \mathbf{e}_{a}\right)
$$

и используя выражение для тензора Риччи через лапласиан и даламбертиан,

$$
\mathbf{R}_{a}=\left(\Delta+\nabla^{2}\right) \mathbf{e}_{a}, \quad \Delta=\delta \mathbf{d}+\mathbf{d} \delta,
$$

где $\mathbf{d}$ - внешний дифференциал, $\delta$ - кодифференциал, $\mathbf{d} \mathbf{d}=\delta \delta=0$, представим уравнения Эйнштейна в квазимаксвелловской форме с сохраняющейся, козамкнутой величиной в правой части:

$$
\delta \mathbf{d e}_{a}=\frac{8 \pi G}{c^{4}} \mathbf{S}_{a}
$$

где

$$
\mathbf{S}_{a}=\mathbf{T}_{a}-\frac{1}{2} T \mathbf{e}_{a}-\frac{1}{\kappa}\left(\nabla^{2} \mathbf{e}_{a}+\mathbf{d} K_{a}\right), \quad \delta \mathbf{e}_{a}=K_{a}, \quad \delta=*^{-1} \mathbf{d} * .
$$

Здесь * обозначает оператор Ходжа, $K_{a}$ - растяжение тетрады, равное нулю для лоренцевой ее калибровки.

Для левых и правых частей уравнений (16) выполняется закон сохранения

$$
\begin{gathered}
\delta \delta \mathbf{d e}_{a} \equiv 0, \quad \delta \mathbf{S}_{a}=-\nabla^{\mu} S_{a \mu}=-\nabla^{\mu}\left(T_{a \mu}+t_{a \mu}\right)=0, \\
t_{a \mu}=\frac{1}{\kappa}\left(-\nabla^{2} e_{a \mu}-\partial_{\mu} K_{a}\right)-\frac{1}{2} T e_{a \mu} .
\end{gathered}
$$

Все величины $\delta \mathbf{d e}_{a}$ тривиальны только в мире Минковского. Более того, этот комплекс отдает предпочтение синхронной системе отсчета как привилегированной ${ }^{2)}$ :

$$
\mathbf{e}_{0}=\mathbf{d} t \quad \rightarrow \quad \delta \mathbf{d} \mathbf{e}_{0} \equiv 0
$$

и даже в случае искривленного пространства-времени плотность энергии $S_{00}$ тождественно равна нулю в соответствии с принципом эквивалентности ${ }^{3)}$.

\footnotetext{
2) Используя синхронно-сопутствующую систему отсчета, можно построить новую теорию квантовой гравитации, и данная теория в настоящий момент находится в разработке.

3) На самом деле $\delta \mathbf{d e} \mathbf{e}_{0} \equiv 0$ для более широкого класса тетрад, а именно для замкнутых $\left(\mathbf{d e} \mathbf{e}_{0}=0\right)$, однако по лемме Пуанкаре всякая замкнутая форма локально точна.
} 
Для тетрады Шварцшильда имеем согласующееся с ньютоновской теорией значение плотности энергии с поправкой, связанной с замедлением времени:

$$
\begin{aligned}
\frac{8 \pi G}{c^{4}} S_{00} & =e_{0}^{\nu}\left(\delta \mathbf{d e}_{0}\right)_{\nu}=-\frac{e_{0}^{\nu}}{\sqrt{-g}} \partial_{\mu}\left[\sqrt{-g} g^{\mu \lambda}\left(\partial_{\lambda} e_{0 \nu}-\partial_{\nu} e_{0 \lambda}\right)\right]= \\
& =-\frac{e_{0}^{0}}{\sqrt{-g}} \partial_{1}\left[\sqrt{-g} g^{11}\left(\partial_{1} e_{00}\right)\right]=\frac{G^{2} m^{2}}{c^{4}\left(1-2 G m / c^{2} r\right) r^{4}} .
\end{aligned}
$$

Для сферически-симметричной невращающейся звезды получаем плотность полной энергии:

$$
\begin{aligned}
S_{00}= & \frac{c^{4}}{8 \pi G} \frac{1}{r^{2}}\left[2 r^{2}\left(\frac{\Phi^{\prime}}{c^{2}}\right)^{2}\left(1-\frac{2 G m}{c^{2} r}\right)+2 r \frac{\Phi^{\prime}}{c^{2}}\left(1-\frac{2 G m}{c^{2} r}\right)-\right. \\
& \left.-\frac{G}{c^{2}}\left(m^{\prime} r-m\right) \frac{\Phi^{\prime}}{c^{2}}+r^{2} \frac{\Phi^{\prime \prime}}{c^{2}}\left(1-\frac{2 G m}{c^{2} r}\right)\right]
\end{aligned}
$$

Подставим $S_{00}$ в интеграл и разложим в ряд до членов порядка $m^{3} / r^{3}$ :

$$
\begin{aligned}
4 \pi \int_{0}^{R} S_{00} \frac{r^{2} d r}{\sqrt{1-2 G m / c^{2} r}} \approx & \int_{0}^{R}\left[-\frac{m^{2} \Phi^{\prime \prime} G}{4 c^{2}}-\frac{m m^{\prime} \Phi^{\prime} G}{2 c^{2}}+\frac{c^{2} r^{2} \Phi^{\prime \prime}}{2 G}+\frac{r^{2}\left(\Phi^{\prime}\right)^{2}}{G}+\right. \\
& \left.+\frac{c^{2} r \Phi^{\prime}}{G}-\frac{r m \Phi^{\prime \prime}}{2}-\frac{r m\left(\Phi^{\prime}\right)^{2}}{c^{2}}-\frac{r m^{\prime} \Phi^{\prime}}{2}-\frac{m \Phi^{\prime}}{2}\right] d r
\end{aligned}
$$

Применим теорию политроп, получим

$$
\begin{aligned}
& \frac{96 \rho_{c}^{2 / 3} K^{7 / 2} \sqrt{\pi G}}{c^{2} \pi G^{2}} \int_{0}^{\xi_{1}} \xi^{3}\left(\theta^{\prime}\right)^{3} d \xi+\frac{32 \rho_{\mathrm{c}}^{1 / 3} K^{4}}{c^{2} \pi G^{2}} \int_{0}^{\xi_{1}} \xi^{4} \theta^{3}\left(\theta^{\prime}\right)^{2} d \xi+ \\
& +\frac{16 \rho_{\mathrm{c}}^{2 / 3} K^{7 / 2} \sqrt{\pi G}}{c^{2} \pi G^{2}} \int_{0}^{\xi_{1}} \xi^{4} \theta^{3}\left(\theta^{\prime}\right)^{2} d \xi+\frac{8 \rho_{\mathrm{c}}^{1 / 3} K^{5 / 2} \sqrt{\pi G}}{\pi G^{2}} \int_{0}^{\xi_{1}} \xi^{2}\left(\theta^{\prime}\right)^{2} d \xi- \\
& \quad-\frac{8 K^{3}}{\pi G^{2}} \int_{0}^{\xi_{1}} \xi^{3} \theta^{3} \theta^{\prime} d \xi-\frac{8 \rho_{\mathrm{c}}^{1 / 3} K^{5 / 2} \sqrt{\pi G}}{\pi G^{2}} \int_{0}^{\xi_{1}} \xi^{3} \theta^{3} \theta^{\prime} d \xi- \\
& -\frac{2 c^{2} K^{3 / 2} \sqrt{\pi G}}{\pi G^{2}} \int_{0}^{\xi_{1}} \xi^{2} \theta^{3} d \xi .
\end{aligned}
$$

Заметим, что в данном выражении уже содержатся члены, описывающие вклад материи, поскольку сохраняющимися величинами как раз являются $\delta \mathbf{d e}{ }_{a}$, а справа в уравнениях (16) стоят все остальные члены из уравнений Эйнштейна.

Вычислим коэффициенты выражения $A \rho_{\mathrm{c}}^{1 / 3}+B \rho_{\mathrm{c}}^{2 / 3}+D$ :

$$
A=6.422065 \cdot 10^{52}, \quad B=-4.488338 \cdot 10^{42}, \quad D=6.088957 \cdot 10^{58} .
$$




\section{8. СРАВНЕНИЕ РЕЗУЛЬТАТОВ}

Вычислим поправку, связанную с тем, что электроны не являются полностью релятивистскими [1]:

$$
\begin{aligned}
x & =\left(\frac{3 \pi^{2} \rho \lambda_{\mathrm{e}}^{3}}{\mu_{\mathrm{e}} m_{\mathrm{u}}}\right)^{1 / 3}, \\
\epsilon_{e} & =\frac{m_{\mathrm{e}} c^{2}}{\lambda_{\mathrm{e}}^{3}} \frac{1}{8 \pi^{2}}\left[x \sqrt{1+x^{2}}\left(1+2 x^{2}\right)-\ln \left(x+\sqrt{x^{2}+1}\right)\right] \approx \\
& \approx \frac{m_{\mathrm{e}} c^{2}}{\lambda_{\mathrm{e}}^{3}} \frac{1}{4 \pi^{2}}\left(x^{4}+x^{2}\right), \quad x \rightarrow \infty .
\end{aligned}
$$

Считается, что член, пропорциональный $x^{4}$, уже учтен, потому что он дает $3 P=$ $P /(\gamma-1)=\epsilon^{\prime}$, и это соответствует внутренней энергии адиабатического газа с уравнением состояния $P=K \rho^{\gamma}$. Таким образом, поправка равна

$$
\begin{aligned}
\Delta E_{\mathrm{int}}= & 4 \pi \int_{0}^{R} \frac{m_{\mathrm{e}} c^{2}}{\lambda_{\mathrm{e}}^{3}} \frac{1}{4 \pi^{2}} x^{2} \frac{r^{2} d r}{\sqrt{1-2 G m / c^{2} r}} \approx \frac{2 \cdot 3^{2 / 3} c^{3} m_{\mathrm{e}}^{2} K^{3 / 2} \sqrt{\pi G}}{m_{\mathrm{u}}^{2 / 3} \mu_{\mathrm{e}}^{2 / 3} \pi^{2 / 3} \rho_{\mathrm{c}}^{1 / 3} G^{2} h} \int_{0}^{\xi_{1}} \xi^{2} \theta^{2} d \xi- \\
& -\frac{8 \cdot 3^{2 / 3} m_{\mathrm{e}}^{2} c K^{5 / 2} \sqrt{\pi G}}{m_{\mathrm{u}}^{2 / 3} \mu_{\mathrm{e}}^{2 / 3} \pi^{2 / 3} G^{2} h} \int_{0}^{\xi_{1}} \xi^{3} \theta^{2} \theta^{\prime} d \xi+ \\
& +\frac{48 m_{\mathrm{e}}^{2} \pi^{1 / 3} \rho_{\mathrm{c}}^{1 / 3} K^{7 / 2} 3^{2 / 3}}{c m_{\mathrm{u}}^{2 / 3} \mu_{\mathrm{e}}^{2 / 3} G \sqrt{\pi G} h} \int_{0}^{\xi_{1}} \xi^{4} \theta^{2}\left(\theta^{\prime}\right)^{2} d \xi=C \rho_{\mathrm{c}}^{-1 / 3}+A \rho_{\mathrm{c}}^{1 / 3}+D,
\end{aligned}
$$

где $C=1.434069 \cdot 10^{53}$, что совпадает со значением, приведенным в [1]. Мы можем

\begin{tabular}{|c|c|c|}
\hline & Метод & $\rho_{c}, \Gamma / \mathrm{cm}^{3}$ \\
\hline & Энергетический метод & $2.635 \cdot 10^{10}$ \\
\hline 1. & Тетрадный комплекс Фролова & $13.594 \cdot 10^{10}$ \\
\hline 2. & Тетрадный комплекс Родичева & $-6.797 \cdot 10^{10}$ \\
\hline 3. & Преимущественный комплекс & $3.195 \cdot 10^{10}$ \\
\hline & Численное интегрирование & $3.555 \cdot 10^{10}$ \\
\hline
\end{tabular}
также вычислить множитель $A=7.937262 \cdot 10^{40}$, но величины коэффициентов $A$ и $D$ не используются в рамках энергетического метода.

Вычисленные по формуле (12) значения $\rho_{\text {c }}$, получившиеся в рамках различных методов, отражены в табл. 2.

ТАБЛИЦА 2

Следовательно, наиболее точный метод из трех приведенных в таблице - это тот, который обозначен номером 3. Это означает, что данный комплекс является "привилегированным". 


\section{9. ЗАКЛЮЧЕНИЕ}

В настоящей статье предложен способ отбора подходящих величин, характеризующих гравитационную энергию в ОТО. Найден привилегированный тетрадный комплекс, который дает значение для центральной плотности белого карлика критической массы, наиболее близкое к значению центральной плотности, полученному при численном интегрировании точных уравнений совместно с близким к точному уравнением состояния. Выяснено, что данный тетрадный комплекс выделяет синхронную систему отсчета как привилегированную для пустого пространства-времени (вакуума). В синхронной системе отсчета полная плотность энергии тождественно равна нулю в соответствии с принципом эквивалентности.

\section{Список литературы}

[1] С. Шапиро, С. Тьюколски, Черные дыры, белье карлики и нейтронные звездь, Т. 1, Мир, М., 1985.

[2] Я. Б. Зельдович, И. Д. Новиков, Теория тяготения и эволюиия звезд, Наука, М., 1971.

[3] Ч. Мизнер, К. Торн, Дж. Уилер, Гравитация, т. 2, Мир, М., 1977.

[4] Ю.С. Владимиров, Системы отсчета в теории гравитации, Энергоиздат, М., 1982.

Поступила в редакцию 6.05.2009 